\title{
The new period determination and mode identification of $\delta$ Scuti star HR 5437
}

\author{
Liu Zong-Li, Zhou Ai-Ying, Jiang Shi-Yang, Liu Yan-Ying, and Li Zhi-Ping \\ Beijing Astronomical Observatory, Chinese Academy of Sciences, 100012 Beijing, China \\ Internet: lzl@class1.bao.ac.cn
}

Received December 4, 1998; accepted March 12, 1999

\begin{abstract}
The $\delta$ Scuti variable star HR 5437 was observed with the $85 \mathrm{~cm}$ telescope at the Xinglong Station of Beijing Astronomical Observatory from 1997 to 1998. Period analyses were made for our data set, the data set obtained by $\mathrm{Li}$ and Jiang in 1991 and the combined data set (1991-1998). From the combined data set two frequencies were obtained: 11.3484 and $6.9840 \mathrm{~cd}^{-1}$. We calculated the evolution sequences of a star with $1.0-2.0$ solar mass and all pulsation modes of each evolution phase. Among all the modes calculated from the 140th evolution phase of a star with 1.90 solar mass we found two frequencies which are almost the same as the two observed frequencies. So, we tend to suggest that HR 5437 pulsates in two modes: a radial p mode with $l=0, n=4$ and another nonradial p modes with $l=1, n=1$.
\end{abstract}

Key words: stars: $\delta$ Scuti — stars: oscillations — stars: individual: HR 5437

\section{Introduction}

HR 5437 is a 6.24 magnitude star situated at $\alpha=$ $14^{\mathrm{h}} 30^{\mathrm{m}} 4, \delta=+60^{\circ} 26$ ! 7 (1950 coordinates). The photoelectric observations of HR 5437 were carried out between 1986 and 1988 at the Piszkéstetö Station of Konkoly Observatory of Hungary and the Xinglong Station of Beijing Astronomical Observatory of China. From the data obtained at the Xinglong Station in 1988 Jiang \& Li (1988) discovered HR 5437 to be a $\delta$ Scuti variable star. But they did not give the values of frequency. From the 1988 data set obtained at the Piszkéstetö Station and the Xinglong Station, Paparó et al. (1990) gave two frequencies. The first frequency is $8.437 \mathrm{~cd}^{-1}$, the second is $12.362 \mathrm{~cd}^{-1}$. Joining the 1988 data set and the data obtained at the Piszkéstetö Station in 1986-1987,

Send offprint requests to: Liu Zong-Li
Paparó et al. (1990) gave two other frequencies. The first is $8.537 \mathrm{~cd}^{-1}$ and the second is $11.376 \mathrm{~cd}^{-1}$. Paparó et al. pointed out that due to the poor quality of the data distribution and the complex structure of the spectral window the precise values of frequencies were not found. Li \& Jiang (1992) obtained only one frequency of $11.390 \mathrm{~cd}^{-1}$ from their data obtained in 1991. However, comparing with the results given by Paparó et al. (1990), Li \& Jiang (1992) thought that the frequency of $8.537 \mathrm{~cd}^{-1}$ given by Paparó et al. (1990) was the fundamental frequency, the frequency of $11.390 \mathrm{~cd}^{-1}$ was the first overtone frequency. The situation is quite confused.

In this paper we wish to make period analyses not only for present data, but also for the data obtained by $\mathrm{Li} \&$ Jiang (1992), and also for the joint data of the two data sets to obtain more accurate frequency solution and investigate the pulsation modes of this star.

\section{Observations}

In order to obtain the correct pulsation frequencies of HR 5437 and study its character we observed this star from 9 to 17 April 1997 and on March 6, 1998 using the $85 \mathrm{~cm}$ telescope at the Xinglong Station of Beijing Astronomical Observatory. The 4-channel photometer (Michel \& Chevreton 1991) with Strömgren $v$ filter was used. The star SAO 16408 was used as comparison, and SAO 16394 was used as check star. The three stars and sky background were observed simultaneously. The integration time was one minute. The value of each data point is the magnitude difference (HR 5437 SAO 16408) normalized to zero. No evidence for any variability of SAO 16408 was found, but SAO 16394 was discovered unexpectedly to be a new $\delta$ Scuti star (Liu et al. 1998). The measuring error is about $3 \mathrm{mmag}$. Seven tracks of data were obtained for HR 5437 from 1997 to 1998 covering a period of 332 days. The observing log is given in Table 1, where time duration is the length 
Table 1. Observing log of HR 5437 (1997-1998)

\begin{tabular}{ccc}
\hline \hline Date & Time duration (day) & Point number \\
\hline 1997.04 .09 & 0.2757 & 344 \\
1997.04 .10 & 0.2826 & 390 \\
1997.04 .12 & 0.2524 & 359 \\
1997.04 .13 & 0.1466 & 212 \\
1997.04 .16 & 0.2034 & 272 \\
1997.04 .17 & 0.1472 & 201 \\
1998.03 .06 & 0.1738 & 280 \\
\hline \hline
\end{tabular}

of observational run (in days) during each night, point number is the amount of data points during each night.

\section{Period analysis}

The period analyses were performed using Hao's program (1991), the program PERIOD (Breger 1990) and PERIOD96 (Sperl 1996). In these programs all parameters of pulsation are obtained using a combination of single-frequency Fourier transforms and multifrequency least squares of brightness residuals (LSR). The detailed procedure of period analyses is referenced in Liu's papers $(1995,1996)$. Besides our data set, we made period analysis for Li and Jiang's data set obtained in 1991 ( $\mathrm{Li} \&$ Jiang 1992) which is the only available data set in the literature. Moreover, we combined the two data sets into one and made period analysis for this long data set so as to obtain a coherent solution. The data points of the combined data set are shown in Fig. 1, where the ordinate is the magnitude difference of HR 5437 and comparison star normalized to zero. The abscissa is the time (+HJD 2448000). All computed results for the three data sets are given in Table 2. In Table 2 the frequency is in cycles per day, the amplitude and standard error are in milli-magnitude, and the phases are normalized to 1. $\sigma$ is the standard errors of fitting.

From Li and Jiang's data set we only confirmed one frequency of $11.390 \mathrm{~cd}^{-1}$. Prewhitening the first frequency, it seems that there is a peak around or larger than $6 \mathrm{~cd}^{-1}$ in the power spectra. Due to the low observational accuracy and less data points the second frequency could not be confirmed. From our data set we obtained two frequencies: 11.3546 and $5.9966 \mathrm{~cd}^{-1}$. However, the value of second frequency is not stable throughout our whole procedure of data processing. While changing the amount of the data points we found that the value of second frequency varied around or larger than $5.9966 \mathrm{~cd}^{-1}$ sometimes. Although the quality of our data set is good, the quantity still is not enough to obtain a satisfactory result. From the combined data set we obtained two frequencies: 11.3484 and $6.9840 \mathrm{~cd}^{-1}$. They can fit the data points very well (see Fig. 1). The power spectra of the combined data set are shown in Fig. 2 together with the spectral window. Power spectrum (01) is obtained from the original data.
The frequency with strongest power is the first frequency of $11.3484 \mathrm{~cd}^{-1}$. Power spectrum (02) is obtained from the residuals (original data minus first frequency). The frequency with strongest power is the second frequency of $6.9840 \mathrm{~cd}^{-1}$. It is very obviously higher than others. So, we think that the two frequencies obtained from the combined data set are reliable.

\section{Identification of evolution phase and pulsation mode}

In order to compute the evolution phase and identify the pulsation modes of HR 5437, we used a standard stellar structure and evolution code which was developed by Luo $(1991,1997)$ and a classical adiabatic oscillation program in which the linear oscillation theory was developed by $\mathrm{Li}(1990,1994)$. Henyey method, the equation of state developed by Luo (1994a, 1994b, 1997) and the latest version of the OPAL opacity tables (Iglesias \& Rogers 1996) were used in the evolution code. And $\mathrm{H}$ and He burning for evolution was considered. First, we used Luo's code to calculate the evolution sequences of a star with $1.0-2.0$ solar mass for an initial composition $X=0.68, Z=0.02$ and the mixing length $\alpha=1.0$. We calculated 11 evolution sequences from 1.0 to $2.0 M_{\odot}$ in step of $0.1 M_{\odot}$. Each evolution sequence composed of 220 evolution phases. Using Li's program $(1990,1994)$ we then calculated all pulsation modes of each evolution phase. Finally, we found two frequencies: $11.3357 \mathrm{~cd}^{-1}$ with radial p mode $(l=0, n=4)$ and $7.0235 \mathrm{~cd}^{-1}$ with nonradial $\mathrm{p}$ mode $(l=1, n=1)$ in the 140th evolution phase of the evolution sequence of a star with $1.9 M_{\odot}$. These two calculated frequencies were the same values as the observed frequencies within the errors. The evolutionary track of a $1.9 M_{\odot}$ star is shown in Fig. 3. The comparison between the observation of HR 5437 and theoretical calculation of a $1.9 M_{\odot}$ star is listed in Table 3, where $\mathrm{C}$ is the calculated value, $\mathrm{O}$ is the value obtained from the observation, $|\mathrm{O}-\mathrm{C}|$ is the deviation between observation and theoretical calculation, $f_{1}$ is the first frequency, and $f_{2}$ is the second frequence. From Table 3 we can see the deviations are very small. It is only about 0.01 for the first frequency and 0.04 for the second one. $l, n$ is the calculated order and degree of a pulsation mode, respectively. So, we tend to suggest that HR 5437 pulsates in two modes: radial $\mathrm{p}$ mode with $l=0, n=4$ and nonradial p mode with $l=1, n=1$.

In order to check the reliability of pulsation modes and evolution we used the absolute bolometric magnitude $M_{\text {bol }}=1.25 \mathrm{mag}$ and effective temperature $T_{\text {eff }}=7900 \mathrm{~K}$ which were derived from the Strömgren $u v b y \mathrm{H}_{\beta}$ photometric data of HR 5437 and used some calibration formulas (Li \& Jiang 1992). And, we know that the absolute bolometric magnitude of the sun $M_{\mathrm{bol}} \odot$ is $4.75 \mathrm{mag}$. Putting $M_{\mathrm{bol}}=1.25 \mathrm{mag}$ and $M_{\mathrm{bol}} \odot=4.75 \mathrm{mag}$ into the basic formula $M_{\mathrm{bol}}-M_{\mathrm{bol} \odot}=-2.5 \log L / L_{\odot}$ we obtained the luminosity of HR 5437 in solar unit $\log L / L \odot=1.40$. 


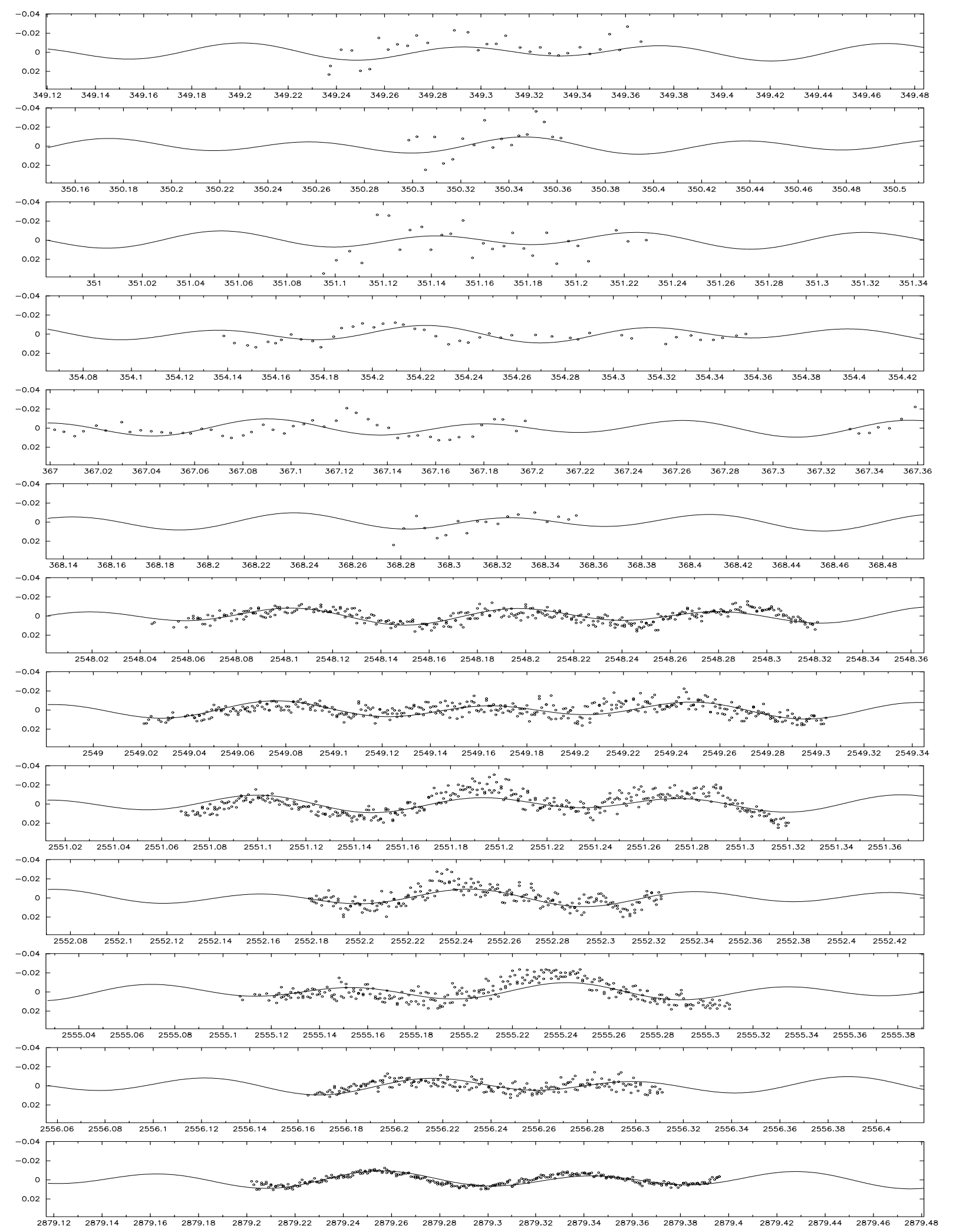

Fig. 1. The data points and fit curves of HR 5437 during 1991-1998. The ordinate is the magnitude difference of HR 5437 and comparison normalized to zero. The abscissa is the time (+HJD 2448000). The fit of the two-frequency solution given in Table 2 is shown as a solid curve 
Table 2. The pulsation frequency solutions of three data sets of HR 5437

\begin{tabular}{llclll}
\hline \hline $\begin{array}{c}\text { Time } \\
(\text { year })\end{array}$ & $\begin{array}{l}\text { Frequency } \\
\left(\mathrm{cd}^{-1}\right)\end{array}$ & $\begin{array}{c}\text { Amplitude } \\
(\mathrm{mmag})\end{array}$ & $\begin{array}{l}\text { Phase } \\
(0-1)\end{array}$ & $\begin{array}{l}\sigma \\
(\mathrm{mmag})\end{array}$ & Data source \\
\hline 1991 & 11.390 & 7.8 & 0.15 & 9.0 & Li \& Jiang (1992) \\
$1997-1998$ & 11.3546 & 7.1 & 0.05 & 6.0 & Present \\
& 5.9966 & 3.0 & 0.65 & & \\
$1991-1998$ & 11.3484 & 6.8 & 0.15 & 6.6 & Li \& Jiang (1992)+Present \\
& 6.9840 & 2.8 & 0.04 & & \\
\hline \hline
\end{tabular}
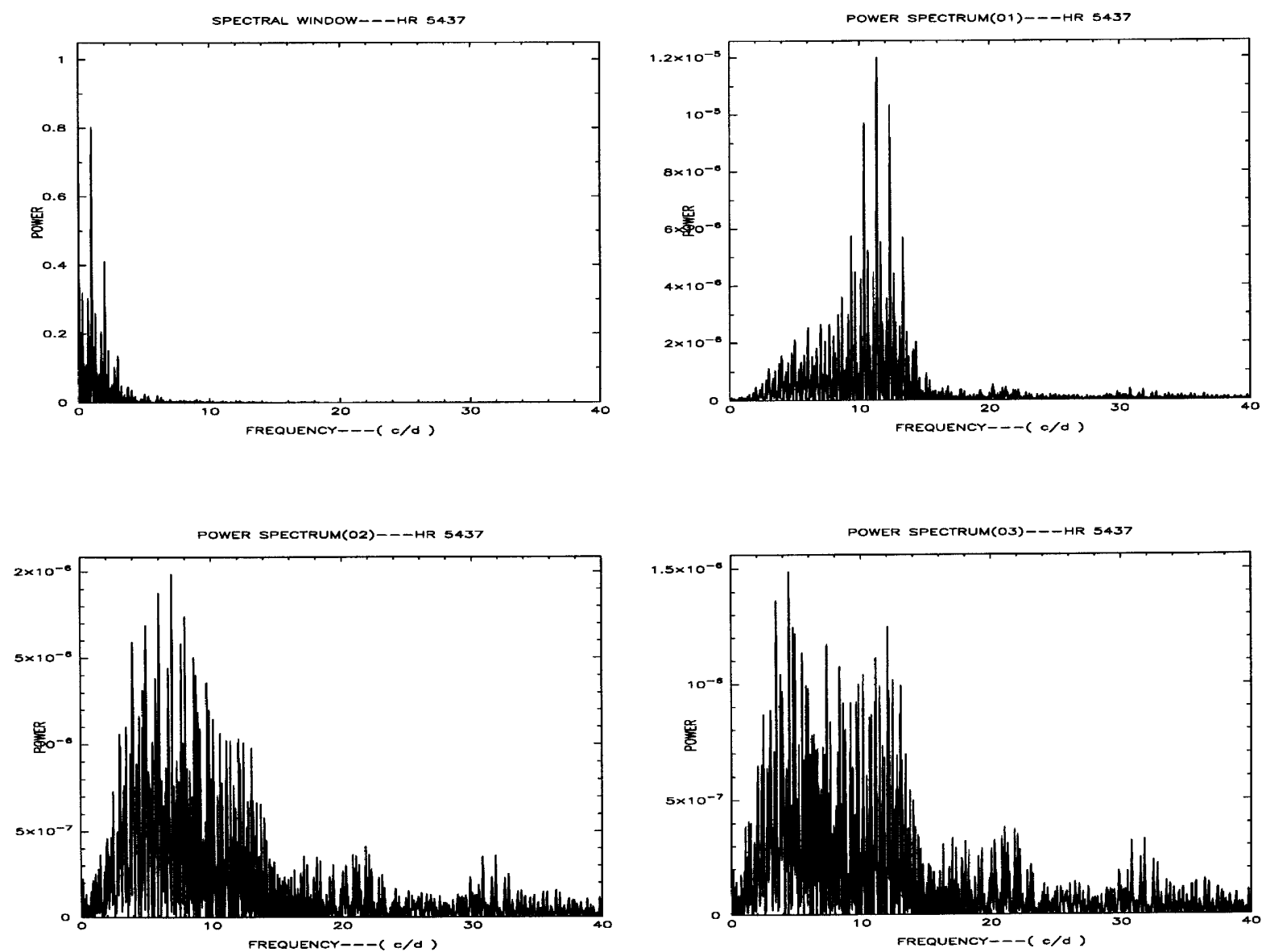

Fig. 2. Power spectra of the 1991-1998 data set of HR 5437. Power spectrum (01) is obtained from the original data. The frequency with strongest power is the first frequency $\left(11.3484 \mathrm{~cd}^{-1}\right)$. Power spectrum (02) is obtained from the residuals (original data minus first frequency). The frequency with strongest power is the second frequency $\left(6.9840 \mathrm{~cd}^{-1}\right)$. Power spectrum $(03)$ is obtained from the residuals (original data minus first and second frequencies). The ordinate is the power $\left(\mathrm{mag}^{2}\right)$. The abscissa is frequency $\left(\mathrm{cd}^{-1}\right)$

Table 3. The comparison of pulsation frequencies and parameters between observation of HR 5437 and theoretical calculation of a star with 1.9 solar mass

\begin{tabular}{ccccccc}
\hline \hline Item & Mode & $l$ & $n$ & $\mathrm{O}$ & $\mathrm{C}$ & $|\mathrm{O}-\mathrm{C}|$ \\
\hline$f_{1}$ & $\mathrm{p}$ & 0 & 4 & 11.3484 & 11.3357 & 0.0127 \\
$f_{2}$ & $\mathrm{p}$ & 1 & 1 & 6.9840 & 7.0235 & 0.0395 \\
$\log \left(L / L_{\odot}\right)$ & & & & 1.40 & 1.41 & 0.01 \\
$\log T_{\text {eff }}$ & & & & 3.90 & 3.90 & 0.0 \\
\hline \hline
\end{tabular}

And the logarithm of effective temperature equals 3.90: $\log T_{\text {eff }}=3.90$. We found that the deviations between these two values and calculated values in the 140th evolution phase of the evolution sequence of a star with $1.9 M_{\odot}$ are small. The comparison between theoretical calculation and observation is listed in Table 3 . The observed position of HR 5437 on the evolution path is shown in Fig. 3 with an asterisk. It places exactly on the modeling evolution sequence curve. 


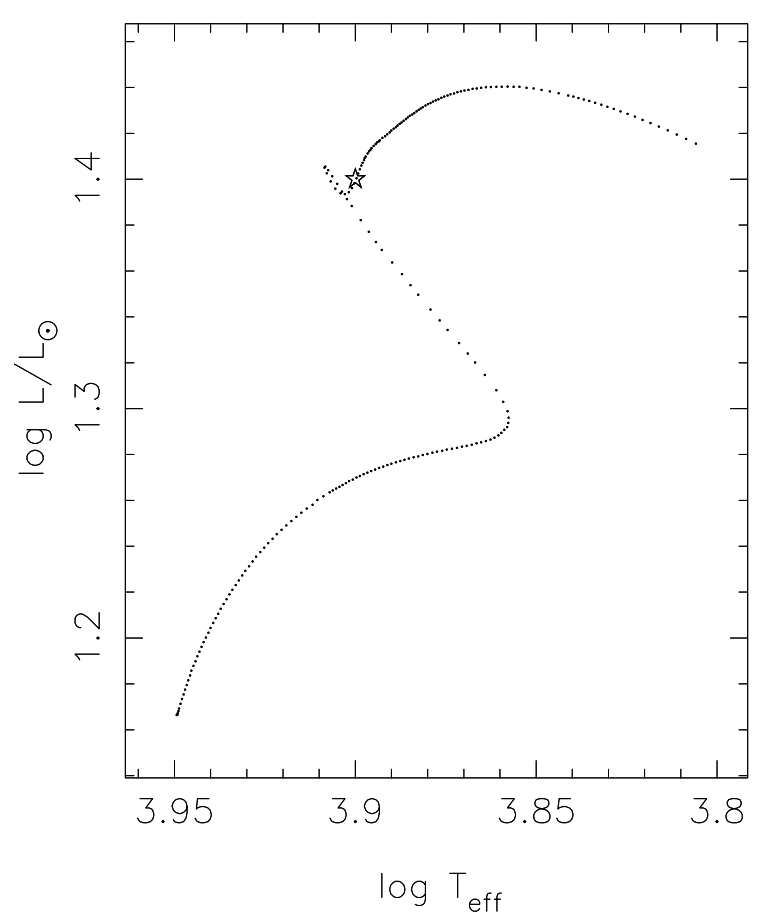

Fig. 3. The standard evolutionary track of the $1.9 M_{\odot}$ model with an initial chemical composition $X=0.68, Z=0.02$. The observed position of HR 5437 is described with an asterisk. The ordinate is luminosity and the abscissa is effective temperature

\section{Conclusion}

As stated above, we made period analyses for three data sets: Li and Jiang's data set, our data set and the combined data set. The main results obtained are given in three tables and three figures. A coherent solution of two frequencies: 11.3484 and $6.9840 \mathrm{~cd}^{-1}$ was obtained. We used the stellar structure and evolution code developed by Luo $(1991,1997)$ and Li's program $(1990,1994)$ to calculate the evolution sequences of a star with $1.0-2.0$ solar mass and all pulsation frequencies of each evolution phase. we found two frequencies: $11.3357 \mathrm{~cd}^{-1}$ (radial p mode, $l=0, n=4$ ) and $7.0235 \mathrm{~cd}^{-1}$ (nonradial $\mathrm{p}$ mode, $l=1, n=1$ ) in the 140th evolution phase of the evolution sequence of a star with 1.9 solar mass. These two calculated frequencies were the same values as the observed frequencies within the errors. So, we tend to suggest that HR 5437 pulsates in the two modes: radial $\mathrm{p}$ mode with $l=0, n=4$ and nonradial $\mathrm{p}$ mode with $l=1, n=1$. Furthermore, from the known parameters: $M_{\mathrm{bol}}=1.25 \mathrm{mag}$ and $T_{\text {eff }}=7900 \mathrm{~K}$ we obtained $\log L / L_{\odot}=1.4$ and $\log T_{\text {eff }}=3.9$. These two parameters are consistent with the calculated values in the 140th evolution phase of the evolution sequence of a star with 1.9 solar mass. It means that the mode identification is reliable.

Acknowledgements. The authors would like to express their thanks to the National Natural Science Foundation of China for the research grants.

\section{References}

Breger M., 1990, Comm. Asteroseismology 20, 1, Austrian Acad. Sciences, Vienna

Hao J.X., 1991, Publ. Beijing Astron. Obs. 18, 35

Iglesias C.A., Rogers F.J., 1996, ApJ 464, 943

Jiang S.Y., Li Z.P., 1988, Astron. Circ. 22, China

Li Y., 1990, A\&A 229, 469

Li Y., 1994, A\&A 286, 815

Li Z.P., Jiang S.Y., 1992, A\&AS 93, 87

Liu Z.L., 1995, A\&AS 113, 477

Liu Z.L., 1996, A\&AS 119, 55

Liu Z.L., Jiang S.Y., Zhou A.Y., et al., 1998, IBVS, No. 4592

Luo G.Q., 1991, Ph.D Thesis, St. Andrews University

Luo G.Q., 1994a, A\&A 284, 679

Luo G.Q., 1994b, A\&A 284, 684

Luo G.Q., 1997, ApJ 491, 366

Luo G.Q., 1997, Chinese J. Comp. Phys. 14, 652

Michel E., Chevreton M., 1991, ASPCS 11, 332

Paparó M., Jiang S.Y., Li Z.P., Kollath Z., 1990, IBVS, No. 3451

Sperl M., 1996, Delta Scuti Star Newslett. 10, 5 